Proceedings of the International School and Conference on Optics and Optical Materials, ISCOM07, Belgrade, Serbia, September 3-7, 2007

\title{
Influence of Laser Pulse Spatial Profile on Optodynamic Source Shape in Liquid Media
}

\begin{abstract}
D. HorvaT ${ }^{a}$ AND M. TERzIĆc ${ }^{b, *}$
${ }^{a}$ Faculty of Mechanical Engineering, University of Ljubljana, Slovenia

${ }^{b}$ Faculty of Science, University of Novi Sad, Serbia

The aim of the present study is to demonstrate the influence of the spatial nonhomogeneity of the laser beam intensity profile on laser induced breakdown in aqueous medium with the use of optodynamic methods. In the experiment the optodynamic waves were induced with pulsed Nd:YAG laser beam which had several "hot spots". The resulting optodynamic transient waves were detected with two detectors: a laser beam deflection probe inside the liquid, parallel to the liquid surface and a piezoelectric transducer at the bottom of the container. The results of the experiment indicate the existence of numerous smaller waves that are observed before the main signal in the optodynamic wave form, contrary to wave forms generated with a laser beam having a smooth spatial profile.
\end{abstract}

PACS numbers: 42.62.Be

\section{Introduction}

The detailed knowledge of the special characteristics of the laser beams propagating through optical systems has an important impact on the success of the applications of laser sources for investigation and treatment of biological media $[1,2]$. The spatially unsmooth laser beam profile can significantly influence the mechanism of laser induced breakdown (LIB) processes. The need to study the parameters which influence the creation of the LIB site has largely grown out of the increasing clinical use of Nd:YAG laser systems for various kinds of microsurgery [3]. For this, and other applications it is important to monitor LIB processes in real time. It was already established that optodynamic (OD) methods of ultrasound detection are suitable for the task $[4,5]$.

*corresponding author; e-mail: mterzic@im.ns.ac.yu 
The spatial as well as the temporal distribution of the laser beam energy is of great importance regarding the complex mechanisms of breakdown and ablation generation [6] as well as OD wave generation. The aim of this work was to investigate this influence experimentally.

\section{Experimental method}

A similar experimental arrangement used for the measurements is described elsewhere [7] and only the relevant details are given here. The excitation laser beam was a multimode Nd:YAG laser beam (1064 nm, pulse width of $10 \mathrm{~ns}$ ) with several "hot spots", with elliptical profile area of $9 \times 16 \mathrm{~mm}^{2}$ and the pulse energy up to $760 \mathrm{~mJ}$. A small portion of the beam was reflected to reach a photodiode that triggered the oscilloscope.

A lens with focal length of $100 \mathrm{~mm}$ was mounted on a micrometric translation stage to vary the position of the focus from positions in the air, on the liquid surface and inside the liquid. The excited OD waves in different liquid samples were registered simultaneously with probe beam deflection method [8-10] and piezoelectric (PE) pressure detection. The investigated liquids were distilled water, tap water, and $\mathrm{CuSO}_{4}$ aqueous solutions, widely used in industry, agriculture, medicine and veterinary medicine. The probe beam, a $10 \mathrm{~mW} \mathrm{He}-\mathrm{Ne}$ laser emitting at $632 \mathrm{~nm}$, passed inside the liquid, parallel to the surface, $63 \mathrm{~mm}$ deep. The OD signals were detected by a bipolar photodetector with $3 \mathrm{MHz}$ band width and digitized by an oscilloscope. The PE transducer was placed at the bottom of the liquid cell at a distance $120 \mathrm{~mm}$ from the liquid surface.

\section{Generation of optodynamic waves in liquids}

When light is absorbed in a media there are several different processes involved, from electronic to optical, thermal and mechanical. They are influenced by the properties of the incoming light pulse such as wavelength, pulse energy, and pulse duration as well as optical, thermal, and mechanical properties of the irradiated media. As the laser light is focused, the local power density may be equal to or higher than the breakdown threshold of the material [11]. This leads to the generation of cavitations bubbles and of the optodynamic waves which propagate outward from the source $[4,12]$. The irradiance values at breakdown threshold for water range from $10^{9}-10^{12} \mathrm{~W} / \mathrm{cm}^{2}[13,14]$. For high pressure differences the wave is a shock wave and propagates with supersonic velocity. In the described experimental conditions the shock wave extends only a few hundred micrometers before it loses enough energy to become an ultrasonic pulse, with central frequency in the range of $1 \mathrm{MHz}$.

The shape and size of optodynamic sources depend on laser beam parameters. It was reported that for a Gaussian incident laser beam, the size of optodynamic source coincides well with the plasma length [12]. A quite different situation can arise when multimode laser beams are used. Non-uniform spatial variations 
of plasma emission induced were observed in air [15]. According to this, more complex behavior of OD waves can be expected.

\section{Results and discussion}

For all investigated liquids, five series of OD waves at different energies (340, $400,520,650$, and $760 \mathrm{~mJ}$ ) were recorded. The beginning of one series starts at focus position marked $0 \mathrm{~mm}$, above the liquid, approaching liquid boundary (at $22 \mathrm{~mm}$ ) to the focus position inside the bulk of the liquid marked with $40 \mathrm{~mm}$. The focus position was moved in steps of 100 or $200 \mu \mathrm{m}$. The wave forms of complete series are put together to form a matrix and presented as a 3D plot. The results for one series of OD wave forms are presented in Fig. 1. Relevant quantities from this measurement were evaluated: wave form amplitude and arrival time of the main signal.
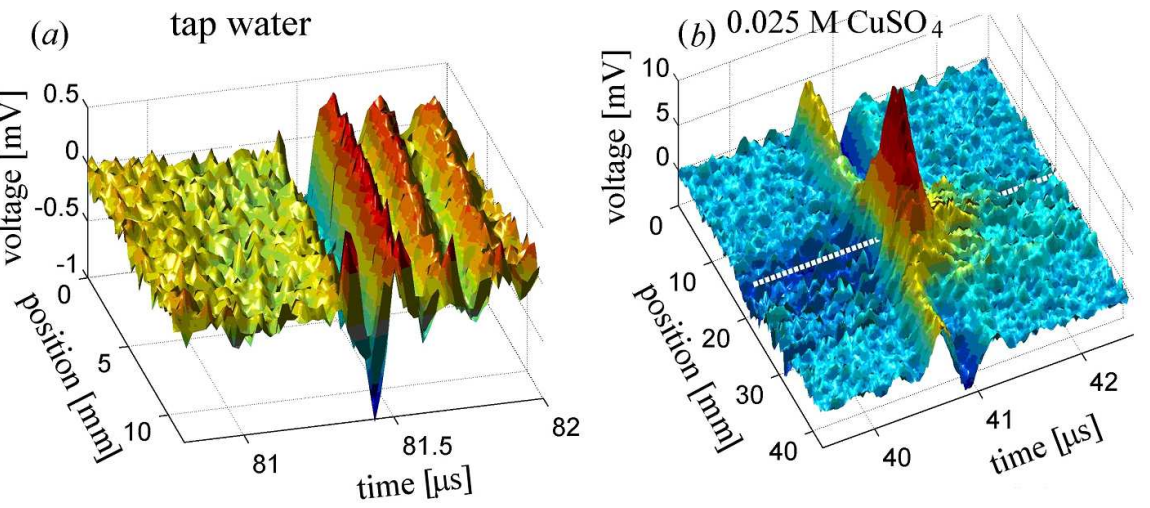

Fig. 1. 3D representation of one series of OD wave forms measured with PE transducer induced in tap water (a), and measured with laser probe in $0.025 \mathrm{M}$ aqueous solution of $\mathrm{CuSO}_{4}$ (b) for $400 \mathrm{~mJ}$, for different laser focus positions.

The wave form amplitude is always greatest when the focus is near the liquid surface, with a nonlinear rise and fall off. The onset of the rise depends on the excitation energy [7].

From the analysis of the arrival time, it was observed that for both detectors and for all investigated liquids, the main signal arrives always around $40 \mu \mathrm{s}$ for the laser deflection probe and $80 \mu \mathrm{s}$ for the PE transducer, irrespective to the position of the pump laser focus and energy. The arrival time in all cases corresponds to the distance between the detector and the liquid surface, the velocity of the ultrasonic pulse being $1500 \mathrm{~m} / \mathrm{s}$ for aqueous samples.

The 3D analysis yields additional information which is hard to notice when observing single wave forms one at a time: the pattern of smaller amplitudes can be observed before the main signal (Fig. 2). The pattern is even more obvious if the time interval is chosen in such a way that the main signal is not included. The 

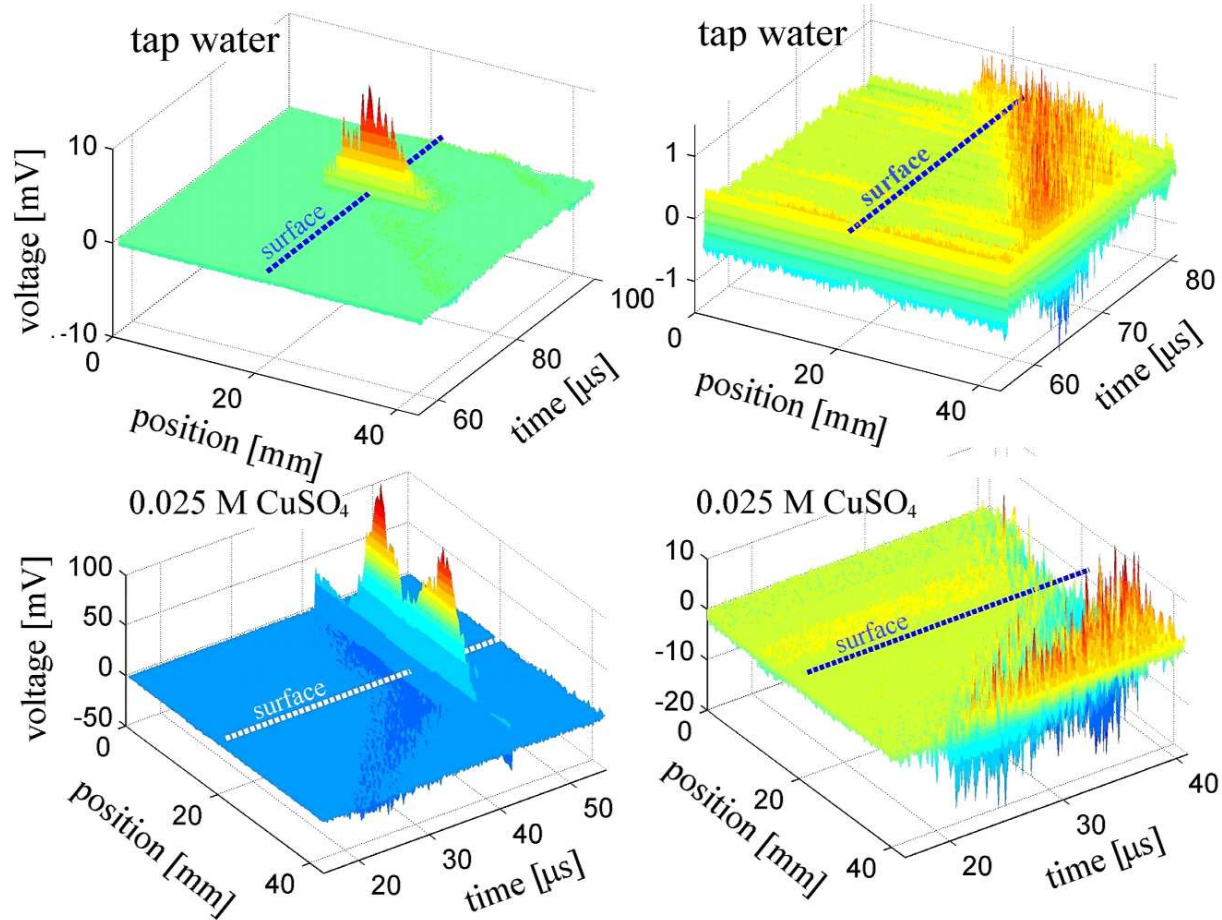

\section{$0.025 \mathrm{M} \mathrm{CuSO}_{4}$}

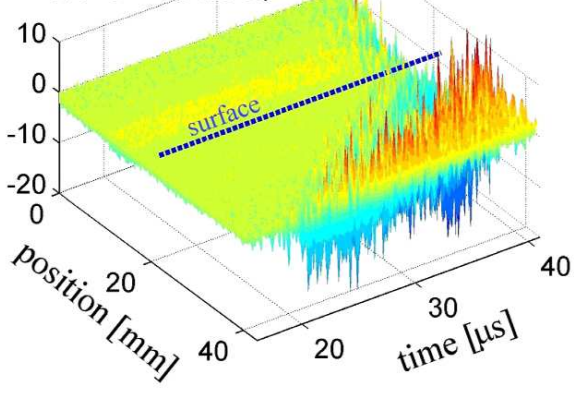

Fig. 2. 3D wave forms plots of the same series: for tap water measured with $\mathrm{PE}$ transducer (upper) and for $0.025 \mathrm{M} \mathrm{CuSO}_{4}$ measured with laser deflection probe (lower). In the left row the main signal is included in the time interval while on the right it is not so that the region of small amplitudes is easily observed.

amplitudes appear to be randomly distributed inside the interval but the beginning and the extent of the interval are not random and depend on the position of the pump laser focus. The maximum length of the interval is about $10 \mu \mathrm{s}$. The trend is such that for focal positions above the liquid surface the beginning of the interval is very near the main signal, within a few $\mu$ s. As the focal position moves inside the liquid, the beginning of the interval moves correspondingly away from the main signal, up to $20 \mu \mathrm{s}$ (Fig. 3). When compared to the data points one can see that the beginning of the interval moves in the same way as the focal point of the excitation laser with respect to the liquid surface. The behavior is the same for all series, all liquids, all energies, and both detectors.

Combining visual observations during the experiment to amplitude analysis, the shape of the OD source can be assumed. We propose that the OD source consists of two parts, the first one is a thin disc at the liquid surface and the second one is a collection of tiny sources around the focal area. The diameters of these two parts are approximately equal to the corresponding diameters of the pump beam, while the length of the second part of OD source depends on focus 

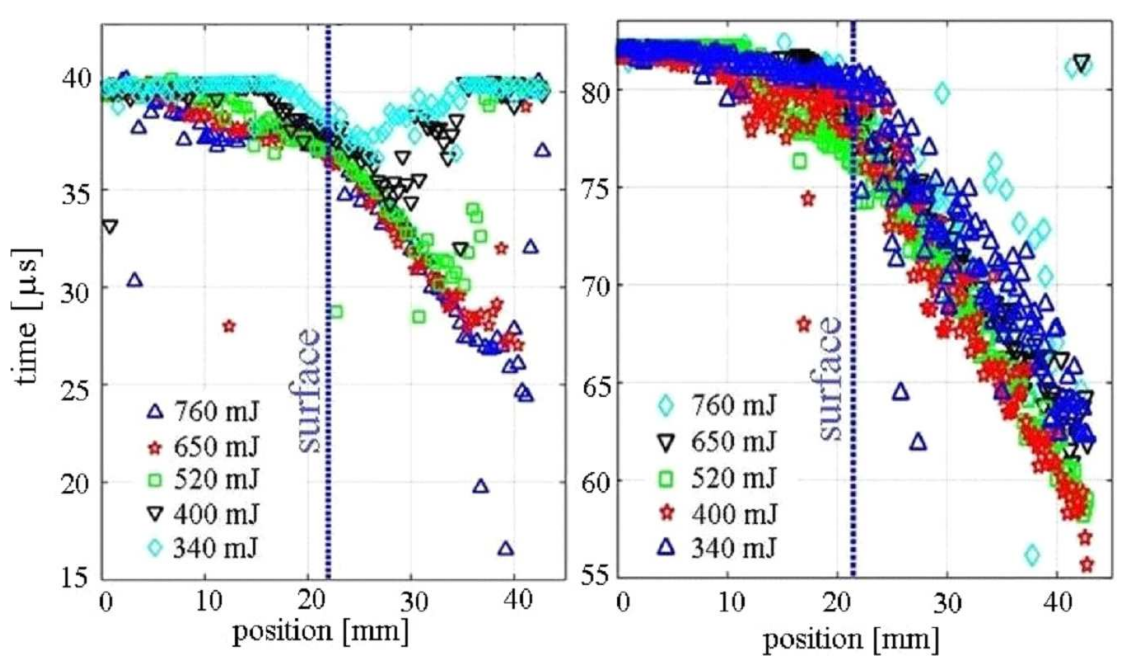

Fig. 3. The beginning time of the interval of small amplitudes as a function of the excitation laser focal position, for different pump energies, $0.05 \mathrm{M}$ solution of $\mathrm{CuSO}_{4}$ (left) and tap water (right) measured with PE transducer.

position relative to liquid surface $(0-20 \mathrm{~mm})$. We believe that the main reason for such a distribution of OD sources is the existence of "hot spots" in the pump beam.

\section{Conclusion}

The effect of complex spatial profile on the laser-liquid interaction has been investigated using optodynamic methods of noncontact ultrasound generation and detection in a transparent liquid. The results presented here suggest that the information about the apparent roughness of the beam spatial profile and the resulting source shape can be deduced by analyzing the OD wave forms. That information can be used to assess the complex shape of the breakdown site which can be useful for clinical applications.

\section{References}

[1] M.H. Niemz, Laser-Tissue Interactions: Fundamentals and Applications, Springer, Netherlands 2004.

[2] A. Vogel, J. Noack, G. Hüttmann, G. Paltauf, J. Phys., Conf. Series 59, 249 (2007).

[3] V. Venugopalan, A. Guerra, K. Nahen, A. Vogel, Phys. Rev. Lett. 88, 078103 (2002).

[4] R. Petkovšek, J. Možina, G. Močnik, Opt. Express 13, 4107 (2005).

[5] D. Horvat, J. Možina, Insight 42, 792 (2000).

[6] E. Tognani, V. Palleschi, M. Corsi, G. Cristoforetti, Spectrochimica Acta B 57, 115 (2002). 
[7] D. Horvat, M. Terzić, J. Možina, Acta Chim Slov. 53, 191 (2006).

[8] J. Diaci, Rev. Sci. Instrum. 63, 5306 (1992).

[9] J. Diaci, J. Možina, Ultrasonics 34, 5306 (1996).

[10] R. Petkovšek, P. Gregorcic, J. Možina, Meas. Sci. Technol. 18, 2972 (2007).

[11] J. Noack, A. Vogel, Appl. Opt. 37, 4092 (1998).

[12] R. Petkovšek, D. Horvat, G. Močnik, M. Terzić, J. Možina, Ultrasonics 44, 266 (2006).

[13] P.K. Kennedy, D.X. Hammer, B.A. Rockwell, Prog. Quant. Electr. 21, 155 (1997).

[14] L.V. Lukin, Chem. Phys. 192, 135 (1995). 